\title{
The Dirac Oscillator in a Rotating Frame of Reference
}

\author{
P. Strange* and L. H. Ryder* \\ School of Physical Science, University of Kent. \\ Canterbury, Kent, CT2 7NH, United Kingdom
}

(Dated: March 23, 2016)

\begin{abstract}
The Dirac equation in a rotating frame of reference is derived from first principles. This equation is employed to exhibit an equivalence between a particle in a Dirac oscillator potential and a free particle in a rotating frame of reference. A zero-point contribution to the energy of the particle, resulting from its spin is also noted.
\end{abstract}

Keywords: Dirac Equation, Dirac oscillator, Rotating frames of reference, free particles. 


\section{INTRODUCTION}

Even non-relativistic quantum theory contains only a very small number of models that can be solved analytically. Models such as the particle in a box, the harmonic oscillator and the hydrogen atom form the basis of our understanding of much of Physics and Chemistry. In relativistic quantum theory the number of exactly soluble problems is even smaller. One such model is the Dirac oscillator[1,2]. This three-dimensional system is defined by the Dirac equation where the momentum operator has an imaginary effective linear vector potential added to it:

$$
\mathbf{p} \rightarrow \mathbf{p}+i m \Omega \beta \mathbf{r}
$$

Here $m$ is the fermion mass, $\Omega$ an oscillator frequency, $\mathbf{r}$ the vector distance of the fermion from the origin of the potential, and $\beta$ is the usual Dirac matrix. As well as being exactly soluble this model has the interesting property that its non-relativistic limit is the usual Schrodinger harmonic oscillator with the addition of strong spin-orbit coupling [3]. For a long time the Dirac oscillator was considered to be an interesting model without applications. However over the last fifteen years the mathematical aspects of this system have been widely studied in one, two and three dimensions [4]-[25]. Additionally, this system has been shown to have an analogy with the (Anti)-Jaynes-Cummings model of quantum optics [26]-[32]. It has also been suggested as a model to describe some properties of graphene [33]. More recently the Dirac oscillator has been found to contain an abrupt chiral phase transition in a magnetic field [34]. This phase transition has been examined in a number of extensions of the model [35] - [39]. After more than 20 years of theoretical activity focussed in the characterization of the Dirac oscillator, a first experimental realization of this system has been developed [40].

In this paper our purpose is to demonstrate a previously unsuspected property of the Dirac oscillator. We demonstrate that there is an equivalence between a particle in the Dirac oscillator potential and a free particle as viewed from a rotating frame of reference.

\section{THE DIRAC EQUATION}

The Dirac equation in a general non-inertial frame in cartesian coordinates has been derived by Hehl and Ni[41] using standard methods [42-44]. Here we follow their procedure 
to derive the Dirac equation in cylindrical polar coordinates in a rotating frame of reference. In its most fundamental form the Dirac equation can be written [44]

$$
i \hbar \gamma^{\lambda}\left(e_{\lambda}+\Gamma_{\lambda}\right) \Psi=m c \Psi
$$

where $e_{\lambda}$ is the coordinate basis determined by the metric, $\Gamma_{\lambda}$ are related to the connection coefficients and $\gamma^{\lambda}$ are the Dirac matrices in the usual representation [3].

The invariant interval in cylindrical polars is

$$
d s^{2}=c^{2} d t^{2}-d r^{2}-r^{2} d \phi^{\prime 2}-d z^{2} .
$$

Introducing a new angular function $\phi$ which is rotating with angular frequency $\omega$ relative to $\phi^{\prime}$

$$
\phi=\phi^{\prime}-\omega t \quad d \phi^{\prime}=d \phi+\omega d t
$$

inserting this into Equation (3), and some manipulation gives [43]

$$
d s^{2}=\left(1-\frac{\omega^{2} r^{2}}{c^{2}}\right)\left(c d t-\frac{1}{c} \frac{r^{2} \omega}{\left(1-\frac{\omega^{2} r^{2}}{c^{2}}\right)} d \phi\right)^{2}-d r^{2}-\frac{r^{2}}{1-\frac{\omega^{2} r^{2}}{c^{2}}} d \phi^{2}-d z^{2}
$$

To derive the Dirac equation it is necessary to write this in terms of basis one-forms $\theta^{j}$. The most convenient way to do this is to employ an anholonomic basis such that

$$
d s^{2}=\theta^{0^{2}}-\theta^{1^{2}}-\theta^{2^{2}}-\theta^{3^{2}}=g_{\mu \nu} d x^{\mu} d x^{\nu}
$$

where $g_{\mu \nu}$ is the metric tensor. The basis one-forms consistent with equations (5) and (6) are

$$
\begin{array}{ll}
\theta^{0}=\left(1-\frac{\omega^{2} r^{2}}{c^{2}}\right)^{1 / 2}\left(c d t-\frac{1}{c} \frac{r^{2} \omega}{\left(1-\frac{\omega^{2} r^{2}}{c^{2}}\right)} d \phi\right) & \theta^{1}=d r \\
\theta^{2}=r\left(1-\frac{\omega^{2} r^{2}}{c^{2}}\right)^{-1 / 2} d \phi & \theta^{3}=d z
\end{array}
$$

It is difficult to proceed without simplification. If it is assumed that $\omega r / c$ can be regarded as a small parameter and linear terms only are retained then

$$
\theta^{0}=c d t-\frac{\omega r^{2}}{c} d \phi, \quad \theta^{1}=d r, \quad \theta^{2}=r d \phi, \quad \theta^{3}=d z
$$

The dual is now defined using

$$
<e_{\nu} \theta^{\mu}>=\delta_{\nu}^{\mu}
$$


yielding a non-coordinate basis

$$
e_{0}=\frac{1}{c} \frac{\partial}{\partial t}, \quad e_{1}=\frac{\partial}{\partial r}, \quad e_{2}=\frac{1}{r} \frac{\partial}{\partial \phi}+\frac{\omega r}{c^{2}} \frac{\partial}{\partial t}, \quad e_{3}=\frac{\partial}{\partial z}
$$

The commutation coefficients are defined by

$$
\left[e_{\mu}, e_{\nu}\right]=C_{\mu \nu}^{\lambda} e_{\lambda}
$$

In our case the only non-zero commutator is

$$
\left[e_{1}, e_{2}\right]=-\frac{1}{r^{2}} \frac{\partial}{\partial \phi}+\frac{\omega}{c^{2}} \frac{\partial}{\partial t}=-\frac{1}{r} e_{2}+\frac{2 \omega}{c} e_{0}
$$

leading to commutation coefficients

$$
C^{2}{ }_{21}=-C^{2}{ }_{12}=\frac{1}{r}, \quad C^{0}{ }_{12}=-C^{0}{ }_{21}=\frac{2 \omega}{c}
$$

All other commutation coefficients are zero. The indices here can be lowered using the metric tensor and then the Christoffel symbols can be found

$$
\Gamma_{\lambda \nu \mu}=\frac{1}{2}\left(C_{\lambda \nu \mu}+C_{\lambda \mu \nu}-C_{\nu \mu \lambda}\right)
$$

The quantities

$$
\Gamma_{\mu}=\frac{1}{8} \Gamma_{\lambda \nu \mu}\left[\gamma^{\lambda}, \gamma^{\nu}\right]
$$

that appear in the Dirac equation can now be calculated.

$$
\Gamma_{0}=\frac{\omega}{4 c}\left[\gamma^{1}, \gamma^{2}\right], \quad \Gamma_{1}=\frac{\omega}{4 c}\left[\gamma^{0}, \gamma^{2}\right], \quad \Gamma_{2}=\frac{1}{4 r}\left[\gamma^{1}, \gamma^{2}\right]-\frac{\omega}{4 c}\left[\gamma^{0}, \gamma^{1}\right]
$$

Putting Equations (10) and (16) into (2) finally gives the explicit form of the Dirac equation in cylindrical polars from the point of view of an observer in a reference frame that is rotating with angular frequency $\omega$ as

$i \hbar\left\{\left(\gamma^{0}+\frac{\omega r}{c} \gamma^{2}\right) \frac{1}{c} \frac{\partial \Psi}{\partial t}+\gamma^{1} \frac{\partial \Psi}{\partial r}+\gamma^{2} \frac{1}{r} \frac{\partial \Psi}{\partial \phi}+\gamma^{3} \frac{\partial \Psi}{\partial z}+\left(\frac{1}{4 r} \gamma^{2}\left[\gamma^{1}, \gamma^{2}\right]-\frac{\omega}{4 c} \gamma^{0}\left[\gamma^{1}, \gamma^{2}\right]\right) \Psi\right\}=m c \Psi$

The wave function is a 4 component quantity $\Psi=\left(\Psi_{1} \Psi_{2} \Psi_{3} \Psi_{4}\right)^{T}$. There are two terms here that contain $\omega$ and hence are introduced by the rotation of the observer's reference frame. Evaluating the commutators shows that the term in $\gamma^{0}\left[\gamma^{1}, \gamma^{2}\right]$ can be written as $-\omega S_{z} / c$ and clearly represents coupling of the particle spin to the rotational motion (Mashhoon effects[45]). The term in $\omega r / c$ includes the angular frequency of rotation, radial distance and the time derivative of the eigenfunction and represents and clearly couples the orbital motion to the particle to the rotation (Sagnac effects[46]). In what follows we will explicitly look at the effect of these terms on the Dirac oscillator. 


\section{THE DIRAC OSCILLATOR IN A ROTATING FRAME OF REFERENCE}

Next, consider the Dirac equation above with the addition of the Dirac oscillator potential according to equation (1). Here we deliberately restrict ourselves to look for solutions that have $\Psi_{2}=\Psi_{3}=0$. Then the coupled differential equations obeyed by the non-zero components of $\Psi$ are

$$
\begin{aligned}
& i \hbar \frac{\partial \Psi_{1}}{\partial t}+\frac{\hbar \omega r}{c} \frac{\partial \Psi_{4}}{\partial t}+i \hbar c \frac{\partial \Psi_{4}}{\partial r}+\frac{\hbar c}{r} \frac{\partial \Psi_{4}}{\partial \phi}+\frac{i \hbar c}{2 r} \Psi_{4}-i m \Omega c r \Psi_{4}-\frac{\hbar \omega}{2} \Psi_{1}=m c^{2} \Psi_{1} \\
& i \hbar \frac{\partial \Psi_{4}}{\partial t}-\frac{\hbar \omega r}{c} \frac{\partial \Psi_{4}}{\partial t}+i \hbar c \frac{\partial \Psi_{1}}{\partial r}-\frac{\hbar c}{r} \frac{\partial \Psi_{1}}{\partial \phi}+\frac{i \hbar c}{2 r} \Psi_{1}+i m \Omega c r \Psi_{1}+\frac{\hbar \omega}{2} \Psi_{4}=-m c^{2} \Psi_{4}
\end{aligned}
$$

To solve these start by assuming a time-dependence of the form

$$
\Psi_{n}(r, \phi, t)=\Psi_{n}(r, \phi) e^{-i W t / \hbar}
$$

where $W$ is the relativistic energy. Then the wave functions can be written

$$
\Psi_{1}(r, \phi)=\psi_{1}(r) \Phi(\phi) \quad \Psi_{4}(r, \phi)=\psi_{4}(r) \Phi(\phi)
$$

and the variables in equation (18) can be separated, leading to

$$
\Phi(\phi)=\exp (i(\mu+1 / 2) \phi)
$$

For the wave function to be continuous this requires that $\mu$ is half-integer. Then the simple

substitutions $\chi_{1}=\psi_{1}$ and $i \chi_{4}=\psi_{4}$ force the radial part of the Dirac equation to become real

$$
\begin{aligned}
& \left(W-m c^{2}-\frac{\hbar \omega}{2}\right) \chi_{1}(r)-\frac{\omega W r}{c} \chi_{4}(r)+\hbar c \frac{d \phi_{4}(r)}{d r}+\frac{\hbar c(\mu+1 / 2)}{r} \chi_{4}(r)-m \Omega c r \chi_{4}(r)=0 \\
& \left(W+m c^{2}+\frac{\hbar \omega}{2}\right) \chi_{4}(r)-\frac{\omega W r}{c} \chi_{1}(r)-\hbar c \frac{d \chi_{1}(r)}{d r}+\frac{\hbar c(\mu+1 / 2)}{r} \chi_{1}(r)-m \Omega c r \chi_{1}(r)=0
\end{aligned}
$$

This equation will be solved in two separate ways.

\section{DIRAC OSCILLATOR-LIKE SOLUTIONS}

Equation (22) makes clear one of the key insights of this paper. The extra term due to the rotation of the reference frame and term describing the Dirac oscillator are both linear 
in $r$ and can be combined into one. Defining an effective frequency

$$
\eta=\left(\frac{W}{m c^{2}} \omega+\Omega\right)
$$

and letting $\chi_{n}=\sqrt{r} \phi_{n}$ equation (22) becomes

$$
\begin{aligned}
& \left(W-m c^{2}-\frac{\hbar \omega}{2}\right) \phi_{1}+\hbar c \frac{\partial \phi_{4}}{\partial r}+\frac{\hbar c}{r}(\mu+1 / 2) \phi_{4}-m c \eta r \phi_{4}=0 \\
& \left(W+m c^{2}+\frac{\hbar \omega}{2}\right) \phi_{4}-\hbar c \frac{\partial \phi_{1}}{\partial r}+\frac{\hbar c(\mu+1 / 2)}{r} \phi_{1}-m c \eta r \phi_{1}=0
\end{aligned}
$$

This is just the radial equation which describes the radial part of the $2+1$ dimensional Dirac oscillator and can be solved in the usual way $[3,48]$. (note that the solution is not valid for the case $\eta=0)$. The final solution is

$$
\Psi(r, \phi, t)=A\left(\begin{array}{c}
\left(\frac{m \eta}{\hbar}\right)^{(\mu+1 / 2) / 2} r^{\mu} L_{n}^{\mu}\left(\frac{m \eta}{\hbar} r^{2}\right) \\
0 \\
0 \\
-\frac{2 i \hbar c}{W+m c^{2}+\hbar \omega / 2}\left(\frac{m \eta}{\hbar}\right)^{(\mu+5 / 2) / 2} r^{\mu+1} L_{n-1}^{\mu+1}\left(\frac{m \eta}{\hbar} r^{2}\right)
\end{array}\right) e^{-m \eta r^{2} / 2 \hbar} e^{i(\mu+1 / 2) \phi} e^{-i W t / \hbar}
$$

where $L_{n}^{\mu}(x)$ are the generalised Laguerre polynomials for principal quantum number $n$ and $A$ is a normalisation constant given by

$$
A=\sqrt{\frac{n !}{\pi \Gamma(n+\mu+1)}}\left(\frac{m \eta}{\hbar}\right)^{1 / 4}\left(1+\frac{4 n m c^{2} \hbar \eta}{\left(W+m c^{2}+\hbar \omega / 2\right)^{2}}\right)^{-1 / 2}
$$

Because $W$ appears in the definition of $\eta$ it is necessary to solve a simple quadratic equation to find an expression for it. Doing this yields

$$
W=2 n \hbar \omega \pm \sqrt{m^{2} c^{4}+m c^{2}(4 n \hbar \Omega+\hbar \omega)+4 n^{2} \hbar^{2} \omega^{2}+\hbar^{2} \omega^{2} / 4}
$$

which reduces to the standard Dirac oscillator expression for $j=l+1 / 2[48]$ when $\omega \rightarrow 0$.

\section{FREE PARTICLE-LIKE SOLUTIONS}

The solutions (25) have the undesirable property that they do not reduce to the standard free particle solutions of the Dirac equation when $\Omega=\omega=0$. Those solutions are written in 
terms of Bessel functions. To remedy this it is necessary to define another effective frequency

$$
\xi=\omega+\frac{m c^{2}}{W} \Omega
$$

and let

$$
\Phi(\phi)=\exp (-i(\mu+1 / 2) \phi)
$$

and following the same procedure as above allows us to write equation (18) as

$$
\begin{array}{r}
\left(W-m c^{2}-\frac{\hbar \omega}{2}\right) \chi_{1}(r)-\frac{\xi W r}{c} \chi_{4}(r)+\hbar c \frac{d \chi_{4}(r)}{d r}+\frac{\hbar c \mu}{r} \chi_{4}(r)=0 \\
\left(W+m c^{2}+\frac{\hbar \omega}{2}\right) \chi_{4}(r)-\frac{\xi W r}{c} \chi_{1}(r)-\hbar c \frac{d \chi_{1}(r)}{d r}+\frac{\hbar c(\mu+1)}{r} \chi_{1}(r)=0
\end{array}
$$

We have been unable to find analytic solutions to these equations in terms of Bessel functions in closed form. However a linear approximation was made in the derivation the Dirac equation which amounted to assuming $\omega r / c<<1$ Assuming $\Omega$ is of the same order as $\omega$ we can write solutions in powers of the small parameter $\xi r / c$. Defining $k_{c}=m c / \hbar$ as the Compton wavevector we find

$$
\begin{aligned}
& \chi_{1}(r)=A J_{\mu+1}\left(k_{c} r\right)+B \frac{\xi r}{c} J_{\mu}\left(k_{c} r\right)+C \frac{\xi^{2} r^{2}}{c^{2}} J_{\mu+1}\left(k_{c} r\right)+\cdots \\
& \chi_{4}(r)=V J_{\mu}\left(k_{c} r\right)+X \frac{\xi r}{c} J_{\mu+1}\left(k_{c} r\right)+Y \frac{\xi^{2} r^{2}}{c^{2}} J_{\mu}\left(k_{c} r\right)+\cdots
\end{aligned}
$$

The next step is to substitute equations (31) into (30) and compare coefficients of $\omega r / c$. Doing this causes the Bessel functions to cancel and we are left with a set of simultaneous equations for the coefficients $A, B, C, V, X$ and $Y$. The determinant associated with these simultaneous equations determines the energy. In principle we can retain as many terms as we wish in equation (31) and the Dirac equation can be solved to arbitrary accuracy. In this case as $c \rightarrow \infty$ only the first term in the expansion (31) remains which is, indeed, the usual solution of the free particle Dirac equation in cylindrical polar coordinates.

\section{DISCUSSION}

There are a number of noteworthy points to make about these calculations. Firstly let us return to equations (23) and (24). In the limit $\Omega \rightarrow 0$ these represent a free particle in a 
rotating frame of reference. However the solutions stay the same and are identical to those of a Dirac oscillator with frequency $W \omega / m c^{2}$. This equivalence between two apparently very different physical systems is the central result of this paper.

Secondly the non-relativistic limit of equation (27) is taken by letting $m c^{2}>>\hbar \Omega$, $\hbar \omega$. In this limit terms of order $\omega^{2}$ can be ignored and the energy becomes

$$
E=W-m c^{2} \approx 2 n \hbar(\omega+\Omega)+\frac{\hbar \omega}{2}
$$

If $\omega=0$ this reduces to the usual expression for the energy of the Dirac oscillator [48]. for the case $j=l+1 / 2$. We made this choice in our expression (21). Clearly in equation (32) $\Omega$ and $\omega$ are treated equivalently in their quantum number dependence. However there is an extra term in $\omega$ in equation (32) that looks like a zero-point energy and there is no corresponding term in $\Omega$. In fact this term arises directly from the fact that particle described has spin 1/2. If we had chosen $\Psi_{1}=\Psi_{4}=0$ and $\Psi_{2}$ and $\Psi_{3}$ non-zero this term would have had the opposite sign. i.e. the direction of rotation of the rotating frame is either parallel or antiparallel to the fermion spin. So if $S_{z}= \pm 1 / 2$ in an inertial frame, the spin in a rotating frame has a different magnitude for the two spin directions. The final term in equation (27) also arise from the spin, but its sign is independent of which pair of components of $\Psi$ are chosen to be non-zero.

The solutions (25) do not reduce to the standard solutions of the free particle Dirac equation when $\omega=\Omega=0[3]$. This leads to the calculation in section which yields a solution in terms of Bessel functions. Here we take the opposite perspective to that taken in the previous section. If $\omega \rightarrow 0$ a particle experiencing the Dirac oscillator potential is equivalent to a free particle as observed from a frame of reference rotating at frequency $\omega=m c^{2} \Omega / W$. We then find the solutions (31) which reduce to the standard free-particle solutions in the non-relativistic limit and as $\omega, \Omega \rightarrow 0$.

Finally we note that even if $\Omega=0$ the eigenfunctions and the energy are quantised. This arises from the fact that if we have a stationary state and cylindrical symmetry the eigenfunction must go to zero as $r \rightarrow \infty$ if they are to have finite energy. This is not unusual and has been found in other cases, [49] for example. 


\section{SUMMARY}

In this paper we have shown a previously unsuspected equivalence between the Dirac oscillator and a free particle as observed from a rotating frame of reference. We have also been that in a rotating frame the spin of the particle results in a zero-point-like term in the energy eigenvalues.

\section{ACKNOWLEDGEMENTS}

One of us (PS) would like to thank K. M. Marshall and P. Weinberger for some helpful comments.

* P.Strange@kent.ac.uk

[1] D. Ito, K. Mori and E. Carrieri, Nuovo Cimento 51A, 1119, (1967).

[2] M. Moshinsky and A. Szczepaniak, J. Phys. A 22, L817 (1989).

[3] P. Strange, Relativistic Quantum Mechanics (Cambridge University Press, Cambridge, England, 1998).

[4] M. Moreno and A. Zentella, J. Phys. A 22, L821 (1989).

[5] J. Benítez, R. P. Martinez y Romero, H. N. Núñez-Yépez, and A. L. Salas-Brito, Phys. Rev. Lett. 64, 1643 (1990).

[6] C. Quesne and M. Moshinsky, J. Phys. A 22, 2263 (1990).

[7] F. Domínguez-Adame and M. A. González, Europhys. Lett. 13, 193 (1990).

[8] J. Beckers and N. Debergh, Phys. Rev. D 42, 1255 (1990).

[9] F. Domínguez-Adame, Phys. Lett. A 162, 18 (1992).

[10] R. P. Martínez-Romero, and A. L. Salas-Brito, J. Math. Phys. 33, 1831 (1992).

[11] V. M. Villalba, Phys. Rev. A 49, 586 (1994).

[12] R. P. Martínez-y-Romero, H. N. Núñez-Yépez, and A. L. Salas-Brito, Eur. J. Phys. 16, 135 (1995).

[13] R. Szmytkowski and M. Gruchowski, J. Phys. A 34, 4991 (2001).

[14] M. H. Pacheco, R. R. Landim, and C. A. S. Almeida, Phy. Lett. A 311, 93 (2003). 
[15] A. D. Alhaidari, Int. J. Theor. Phys. 43, 939 (2004).

[16] N. Ferkous and A. Bounames, Phys. Lett. A 325, 21 (2004).

[17] C. Quesne and V. M. Tkachuk, J. Phys. A 38, 1747 (2005).

[18] C. Quesne and V. M. Tkachuk, J. Phys. A 39, 10909 (2006).

[19] R. Rekioua and T. Boudjedaa, Eur. Phys. J. C 49, 1091 (2007).

[20] R. de Lima Rodrigues, Phys. Lett. A 372, 2587 (2008).

[21] E. Sadurní, J. M. Torres, and T. H. Seligman, J. Phys. A 43, 285204 (2010).

[22] B. P. Mandal and S. Verma, Phys. Lett. A 374, 1021 (2010).

[23] K. Bakke and C. Furtado, Phys. Lett. A 376, 1269 (2012).

[24] Y. Li, K. Intriligator, Y. Yu, and C. Wu, Phys. Rev. B 85, 085132 (2012)

[25] K. Bakke, Gen. Relativ. Gravit. 45, 1847 (2013).

[26] A. Bermudez, M. A. Martin-Delgado, and E. Solano, Phys. Rev. A 76, 041801(R) (2007).

[27] A. Bermudez, M. A. Martin-Delgado, and E. Solano, Phys. Rev. Lett. 99, 123602 (2007).

[28] A. Bermudez, M. A. Martin-Delgado, and A. Luis, Phys. Rev. A 77, 033832 (2008).

[29] A. Bermudez, M. A. Martin-Delgado, and A. Luis, Phys. Rev. A 77, 063815 (2008).

[30] Y. Luo, Y. Cui, Z. Long, and J. Jing, Int. J. Theor. Phys. 50, 2992 (2011).

[31] A. Jellal, A. El. Mouhafid, and M. Daoud, J. Stat. Mech. 01, P01021 (2012).

[32] A. Boumali and H. Hassanabadi, Eur. Phys. J. Plus. 128: 124 (2013).

[33] C. Quimbay and P. Strange, ArXiv: 1311.2021, (2013).

[34] C. Quimbay and P. Strange, ArXiv: 1312.5251, (2013).

[35] J. A. Sanchez-Monroy and C. Quimbay, Annals of Physics, 350 69-83, (2014).

[36] D. Nath and P. Roy, Annals of Physics 351, 13-21, (2014).

[37] O. Panella and P. Roy, Phys. Rev. A 90, 042111, (2014).

[38] L. Menculini, O. Panella and P. Roy, Phys. Rev. D, 91, 045032, (2014).

[39] A. Bournalli and H. Hassanabadi, ArXiv: 1501.07041, (2015).

[40] J. A. Franco-Villafane, E. Sadurni, S. Barkhofen, U. Kuhl, F. Mortessagne, and T. H. Seligman, Phys. Rev. Lett. 111, 170405 (2013).

[41] F. W. Hehl and W. Ni, Phys. Rev D, 42, 2045, (1990).

[42] R. U. Sexl and H. K. Urbantke, Gravitation and Kosmologie, Mannheim: Bibliographisches Institut., (1983). 
[43] W. Rindler, Relativity Special. General and Cosmological, 2nd edition, (Oxford University Press, Oxford, UK) (2006).

[44] L. H. Ryder, Introduction to General Relativity, (Cambridge University Press, Cambridge, UK), (2009).

[45] B. Mashhoon, Physics Letters A, 173, 347, (1993).

[46] G. Sagnac, Comptes Rendues de l'Academie des Sciences, 157, 708 and 1410, (1913).

[47] A. Bermudez, M. A. Martin-Delgado, and A. Luis, Phys. Rev. A. 77, 063815 (2008).

[48] R. Szmytkowski and M. Gruchowski, J. Phys. A, 34, 4991-4997, (2001).

[49] P. Strange, Phys. Rev. A, 89, 044101, (2014). 